\title{
Navigating issues of related submission and embargo
}

Nature Neuroscience clarifies journal policies on overlapping or concurrent submissions and embargo.

A cademic science has always been a competitive endeavorattempting to generate data, publish and publicize your results before others beat you to the punch-but today the stakes are higher than ever. Publishing impactful papers with regularity is an important factor in determining whether a lab will retain (or acquire) funding, particularly in the current environment of ever-shrinking research budgets and increasing operating costs. In addition, the number and quality of your publications can be the determining factor in obtaining tenure or finding a faculty position. Labs with large, multidimensional datasets therefore commonly chop these findings into two or more separate publications to try and maximize the return for all parties involved; moreover, it is not unusual for competitors to seek cosubmission and co-publication in the same journal. Furthermore, the story often does not end with the publication of the paper; increasingly often, researchers and their institutes seek to increase the visibility of their work through press releases and discussions with journalists. However, the proper way to handle these issues of overlapping or concurrent submissions is not fully understood by many laboratories. Similarly, the rules that govern publicizing your work and honoring the embargo date is not always clear. We attempt to clarify our policies regarding these issues below.

Nature Neuroscience has very strict policies regarding the submission of studies describing the same or overlapping datasets or findings to multiple journals. As stated in our Guide to Authors, submission to our journal implies "...that there is no significant overlap between the submitted manuscript and any other papers from the same authors under consideration or in press elsewhere." Thus, it is essential that you alert the editors of any associated or related studies that have been submitted to or accepted (but not yet published) by other journals. Specifically, we ask that, when uploading the primary files for the paper that you are submitting to Nature Neuroscience, you also include a copy of the submitted or accepted related manuscript. In addition, you should describe in your cover letter the focus of this related paper and how it relates to (and, most importantly, how it differs from) the current submission so that we can evaluate the precise overlap between the studies. If your paper is sent out for peer review, the related manuscript will also be made available to the referees, and we will usually ask them to comment specifically on the overlap between the two papers. Failure to provide any related studies at the time of submission could lead to delays in the processing of your manuscript or even rejection of your paper.
Another source of confusion among authors is the policy regarding co-submission of related studies from two or more research groups. Many of us have had the experience where, while attending a meeting, we see a talk or poster describing results that are very similar or even identical to our own. Once each team recovers from the initial bout of panic, it is not uncommon for researchers to agree to co-submit the manuscripts to the same journal so as to avoid having one study scoop the other. However, if you do decide to take this approach, it is important that you keep the following points in mind. First, although we will do our best to give the two papers an equivalent evaluation-ensuring that the papers are held to the same editorial standardsv and, if the papers are sent for peer review, attempting to assign the same referees to the manuscripts-journal policy dictates that each study must be able to stand on its own merits and cannot be buoyed by the strengths of the accompanying paper. Thus, it is theoretically possible for one paper to be accepted and the other rejected; the studies do not necessarily 'live or die' together. In addition, it should be noted that, if both papers are accepted, we will do our best to publish them simultaneously, but this may not always be possible.

While the main audience for any scientific study is generally your peers and colleagues, many researchers are encouraged to disseminate their results to the general public as well. Research institutes and universities often employ press officers whose main job is to act as a liaison between the researchers and the media. However, most journals have explicit rules for when your pre-publication results can be publicized in mass and social media-although scientists have complete liberty in presenting their work at most conferences and symposia and are free to present their work in preprint archives (however, please check with your editor before presenting your work at a conference which publishes full-manuscript proceedings that have been vetted for novelty, such as NIPS Proceedings). Announcement of the results of a soon-to-be-published study to the general public must be postponed until the embargo date, which usually coincides with the online publication of the paper. We urge researchers to communicate with their institutes' press offices and the editors to ensure that any press releases or other publication or broadcast of journalistic articles do not break the journal's embargo.

Given the frequency with which authors and referees query us about these issues, it is clear that navigating the ever-changing landscape of scientific publishing can be confusing. We hope that this editorial clarifies some of these points. When in doubt about these or any other journal-related points, however, authors are urged to contact the editors and ask for clarification. 